\title{
Perfil do cirurgião dentista das equipes de saúde bucal e inserção em ações de educação permanente na Região Norte do Brasil
}

\author{
Dentist profile in the oral health teams and insertion in permanent education actions in the \\ brazil northern region
}

\author{
Danielle Tupinamba Emmi ${ }^{1}$ \\ Orcid: https://orcid.org/0000-0002-6046-0717
}

\author{
Zaryff Said de Lima ${ }^{2}$ \\ Orcid: https://orcid.org/0000-0002-3394-3561
}

\author{
Mayara Sabrina Luz Miranda ${ }^{3}$ \\ Orcid: https://orcid.org/0000-0003-4749-1698
}

\begin{abstract}
Resumo
Introdução: A Educação Permanente em Saúde (EPS) pode transformar e qualificar a atenção à saúde, devendo acontecer cotidianamente, a partir dos problemas apresentados nas instituições. Objetivo: Conhecer o perfil profissional do cirurgião dentista (CD) que atua nas Equipes de Saúde Bucal (ESB) na região Norte do Brasil, e analisar em quais ações de EPS esse profissional se insere. Metodologia: Trata-se de um estudo descritivo com análise documental, com dados coletados do $2^{\circ}$ ciclo do Programa de Melhoria do Acesso e da Qualidade da Atenção Básica (PMAQ-AB), módulo VI, de 2014, com 1.189 CD atuantes nas ESB na região Norte do Brasil. Resultados: A maioria dos CD não apresentava pós-graduação e tinha até 3 anos de atuação na $\operatorname{ESB}(74,3 \%)$. A maioria ingressava no serviço por concurso público $(42,4 \%)$, apesar de a "indicação" (30,6\%) ser a forma predominante nos estados do Amapá $(55,9 \%)$, Amazonas $(35,6 \%)$ e Pará $(38,9 \%)$. Predominantemente tinham vínculo temporário (48,1\%), em que o estado do Amapá se destacou pela presença de $83,1 \%$ de seus CD nesse vínculo. A maioria participava de ações de EPS $(69,6 \%)$, com destaque para os Estados do Amazonas (79,8\%) e Tocantins (74,7\%). Dentre as ações de EPS que o CD se inseria, destacaram-se as atividades presenciais $(85,2 \%)$ e as trocas de experiências $(33,3 \%)$. Conclusão: Constatou-se que o tipo de vínculo do $\mathrm{CD}$ pode gerar instabilidade profissional e pouca interação com o sistema de saúde. As novas estratégias e tecnologias da informação ainda são pouco exploradas como métodos para EPS na Região Norte.
\end{abstract}

Palavras-chave: educação permanente; estratégia saúde da família; saúde pública; políticas públicas

\begin{abstract}
Introduction: Permanent Health Education (EPS) can transform and qualify health care, and should happen on a daily basis, based on the problems presented in the institutions. Objective: To know the professional profile of the dental surgeon (DDS) who works in the Oral Health Teams (ESB) in the North region of Brazil, and to analyze in which EPS actions this professional is inserted. Methods: This is a descriptive study with documental analysis, with
\end{abstract}

\footnotetext{
${ }^{1}$ Faculdade de Odontologia - Universidade Federal do Pará. E-mail: dtemmi@yahoo.com.br

${ }^{2}$ Faculdade de Odontologia - Universidade Federal do Pará. E-mail: zaryffsaid87@gmail.com

${ }^{3}$ Faculdade de Odontologia da Universidade Federal do Pará. E-mail: zaryffsaid87@gmail.com
} 
data collected from the 2nd cycle of the Program for Improving Access and Quality of Basic Care (PMAQ-AB), module VI, of 2014, with 1,189 DDS working in the ESB in the North region of Brazil. Results: Most of the DDS didn't have postgraduate studies and had up to 3 years of work in the ESB (74.3\%). Most entered the service by public tender (42.4\%), although the "indication" (30.6\%) is the predominant form in the states of Amapá (55.9\%), Amazonas (35.6\%) and Pará (38.9\%). Predominantly they had temporary link $(48,1 \%)$, where the state of Amapá stood out for the presence of $83,1 \%$ of its DDS in this link. The majority participated of EPS actions $(69,6 \%)$, with highlight to the States of Amazonas $(79,8 \%)$ and Tocantins $(74,7 \%)$. Among the EPS actions that the DDS was included, there were presential activities $(85.2 \%)$ and exchanges of experiences (33.3\%). Conclusion: It was observed that the type of relationship of the DDS can generate professional instability and little interaction with the health system. New strategies and information technologies are still little explored as methods for EPS in the North Region.

Keywords: permanent education; family health strategy; public health; public policy

\section{Introdução}

A Estratégia Saúde da Família (ESF) constitui-se em uma proposta de reorientação da Atenção Básica à saúde no Brasil, que visa promover a saúde da população e garantir o direito de acesso, equânime e integral, de todos aos serviços de saúde, de acordo com a Constituição Federal de $1988^{1}$. Para isso, é necessário que os profissionais da ESF tenham perfil e conhecimento adequado para suas atribuições, adquiram novas habilidades e competências para atuar na diversidade de demandas e realidades do território adscrito $^{2}$.

Para a saúde bucal esse contexto não é diferente, pois com a implementação da Política Nacional de Saúde Bucal (PNSB), Brasil Sorridente, o cirurgião dentista passou a ter atribuições para melhorar o estado de saúde da população, mediante a construção de um modelo assistencial de atenção baseado na promoção, proteção, diagnóstico precoce, tratamento e recuperação da saúde, de acordo com os princípios e diretrizes do Sistema Único de Saúde (SUS), dirigido ao indivíduo, à família e à comunidade ${ }^{3}$, além de exigir do profissional uma interação com a equipe de saúde de forma integrada, algo bem diferente da prática individual, curativa e centrada na doença, que permeava a odontologia até então.

Para isso, o Ministério da Saúde (MS) propôs que o próprio cenário de práticas cotidianas em saúde fosse visto como local de ensino-aprendizagem pelos trabalhadores, instituindo em 2004, por meio da Portaria GM/MS nº198, a Política Nacional de Educação Permanente em Saúde (PNEPS) ${ }^{4}$, que em 2007 foi complementada pela Portaria GM/MS $\mathrm{n}^{\mathrm{o}} 1996$ estabelecendo novas diretrizes e estratégias para a PNEPS $^{5}$.

A Educação Permanente em Saúde (EPS) objetiva a produção de conhecimentos a partir da realidade vivida nas instituições de saúde pelos atores envolvidos, tendo os problemas enfrentados no dia a dia do trabalho e as experiências desses atores como base de educação e gatilho para a transformação ${ }^{6}$. A EPS exige ferramentas que busquem a reflexão crítica sobre as práticas do serviço, sendo por si, uma prática educativa que possibilita transformações nas relações, nos processos de trabalho, nas condutas, nas atitudes, nos profissionais e até mesmo na equipe ${ }^{7}$.

Esse contexto faz de seus trabalhadores protagonistas do cotidiano dos serviços de saúde, exigindo maior capacidade de análise, intervenção e autonomia para o estabelecimento de práticas transformadoras, bem como a gestão de mudanças e o estreitamento dos elos entre concepção e execução do trabalho para melhoria da qualidade da atenção em saúde.

Contudo, a existência de modelos formativos conservadores e processos de trabalho do cirurgião dentista que não estão 
de acordo com as mudanças do modelo trazidas pelo SUS, dificultam o entendimento da EPS como política de saúde e a adoção das práticas como ferramenta efetiva para reorientação do processo de trabalho na Atenção Primária à Saúde (APS) em vistas à qualidade do serviço ${ }^{8}$.

Nesse enfoque, o Programa de Melhoria do Acesso e da Qualidade da Atenção Básica (PMAQ-AB) instituído pela Portaria GM/MS nº1654, de 19 de julho de $2011^{9}$, visa induzir a ampliação do acesso e a melhoria da qualidade da atenção básica, promovendo a inovação na gestão, fortalecendo os processos de autoavaliação, monitoramento e avaliação; apoio institucional e educação permanente nas três esferas de governo ${ }^{10}$. Observa-se, portanto, que o PMAQ-AB está intimamente ligado à EPS, quando essas ações visam transformar as práticas do fazer, do trabalho e do serviço para melhor enfrentar os desafios e solucionar problemas da comunidade e do território ${ }^{11}$.

O PMAQ-AB é um processo contínuo e visa desconstruir o viés negativo da avaliação, estimulando uma consciência permanente de análise e tomada de decisão para superar problemas e alcançar objetivos pactuados pelas equipes, sendo assim, pode ser considerado um disparador para a pactuação da prática de EPS nas equipes participantes do Programa ${ }^{11}$.

Nesse contexto, a Estratégia Saúde da Família constitui um importante espaço para a consolidação da EPS e conhecer o perfil dos profissionais atuantes nas equipes de saúde assume considerável importância, visto a reformulação das práticas tradicionais em saúde que devem ser praticadas pelos profissionais das equipes. Ademais, muitas dessas informações são incompletas $^{12}$ não abrangendo todos os estados brasileiros.

Dessa forma, considerando a importância do PMAQ como programa indutor de mudanças e que, após 10 anos da instituição da PNEPS ocorreu o $2^{\circ}$ ciclo do PMAQ-AB (2014), o objetivo desta pesquisa foi analisar o perfil do Cirurgião Dentista que atua nas Equipes de Saúde Bucal na Região Norte do Brasil, com base nos dados do $2^{\circ}$ ciclo do PMAQ-AB, analisando comparativamente entre Estados da Região, em quais ações de Educação Permanente este profissional está inserido.

\section{Metodologia}

A presente investigação trata-se de um estudo descritivo com análise documental, a partir de dados secundários do $2^{\circ}$ ciclo do PMAQ-AB, oriundos de banco de dados público, disponibilizados pelo Ministério da Saúde, no Portal da Secretaria de Atenção Primária à Saúde (https://aps.saude.gov.br/ape/pmaq/ciclo2/) , não havendo a necessidade de aprovação do Comitê de Ética em Pesquisa.

Para o estudo foram coletadas informações referentes ao módulo VI, do Instrumento de Coleta da Avaliação Externa do $2^{\circ}$ Ciclo do PMAQ-AB, realizado no ano de 2014.

O módulo VI compreendeu entrevista com o profissional da Equipe de Saúde Bucal (ESB), com o objetivo de obter informações sobre o processo de trabalho da equipe e a organização do serviço e do cuidado aos usuários ${ }^{13}$.

Durante a entrevista, qualquer profissional da ESB (Cirurgião-dentista, Auxiliar em saúde bucal ou Técnico em saúde bucal) poderia responder o questionário, estando condicionada àquele que agregasse o maior conhecimento sobre o processo de trabalho da equipe ${ }^{14}$. Embora 26 Técnicos em Saúde Bucal (TSB) e 80 Auxiliares em Saúde Bucal (ASB) pertencentes à ESB terem respondido a esse módulo durante o processo de Avaliação Externa do $2^{\circ}$ ciclo do PMAQ-AB, para coleta de dados nesta pesquisa foi levado em consideração apenas os módulos respondidos por cirurgiões dentistas (CD) para atender o objetivo desta investigação, totalizando 1.189 cirurgiões dentistas atuantes nas ESB, nos sete Estados da Região Norte do Brasil (Quadro 1). 
Quadro 1: Quantidade de Cirurgiões Dentistas atuantes na ESB nos estados da Região Norte do Brasil, 2014.

\begin{tabular}{|c|c|}
\hline Estado & $\mathbf{N}^{\mathbf{0}}$ de $\mathbf{C D}$ \\
\hline Acre (AC) & 60 \\
\hline Amapá (AP) & 59 \\
\hline Amazonas (AM) & 267 \\
\hline Pará (PA) & 391 \\
\hline Rondônia (RO) & 135 \\
\hline Roraima (RR) & 20 \\
\hline Tocantins (TO) & 257 \\
\hline Total de CD (Região Norte) & 1.189 \\
\hline
\end{tabular}

Os eixos do módulo VI, que tiveram as variáveis de pesquisa analisadas foram: 1) Informações gerais sobre o entrevistado (VI.2); 2) Formação e qualificação do CD (VI.3) 3) Vínculo (VI.4); 4) Plano de carreira (VI.5); e 5) Educação permanente no processo de qualificação das ações desenvolvidas $\left(\right.$ VI.6) ${ }^{14}$.
Para análise de dados foi verificada a frequência absoluta e relativa de cada variável em cada um dos sete estados da Região Norte, utilizando planilha do Microsoft Word Excell, versão 2010, sendo os resultados expressos em tabelas e gráficos.

Gráfico 1: Participação dos CD nas ações de EPS. PMAQ-AB, 2014.

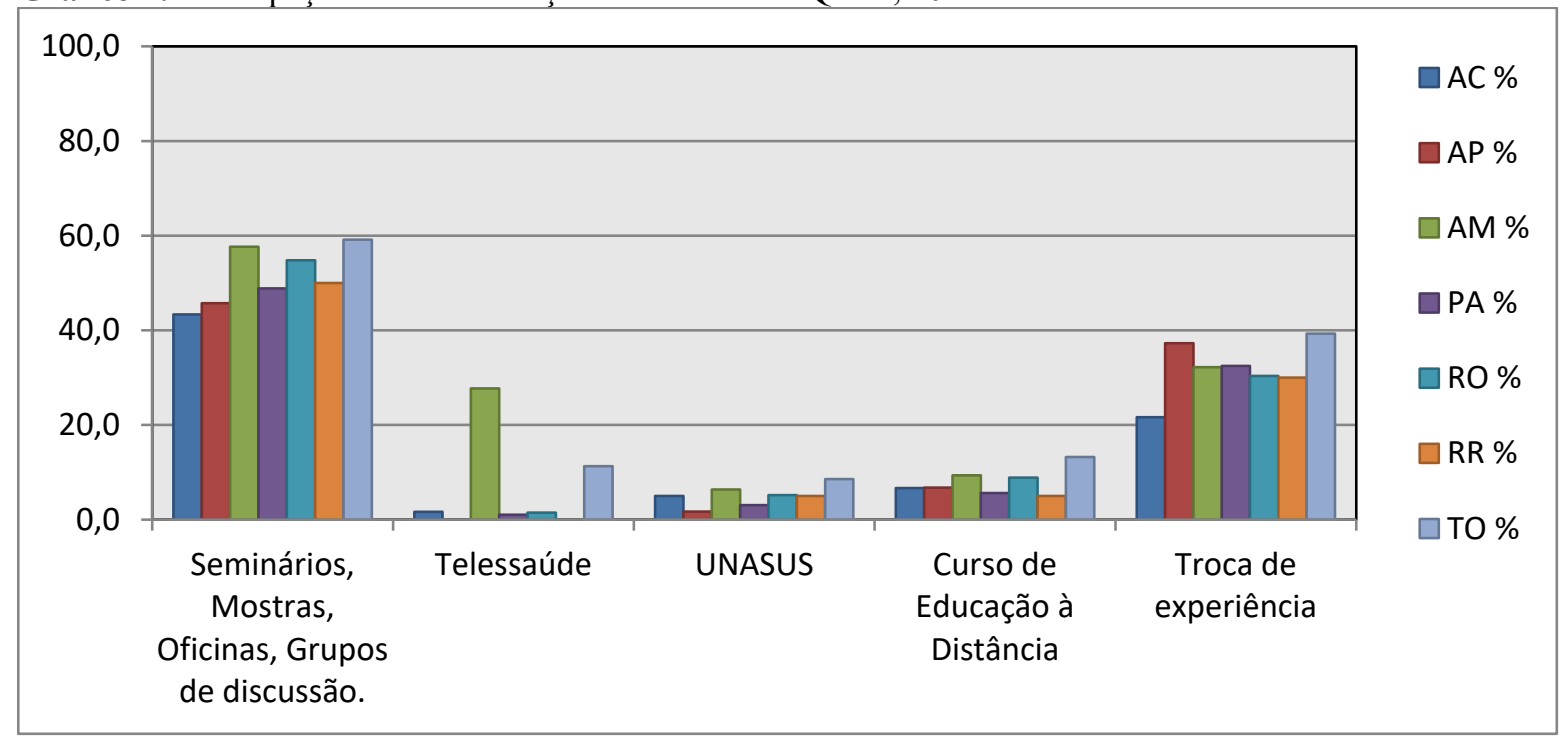

Fonte: PMAQ-AB, 2014.

\section{Resultados}

Observou-se que os Estados do Pará (391), Amazonas (267) e Tocantins (257) apresentaram o maior número de $\mathrm{CD}$ participantes da fase de avaliação externa do $2^{\circ}$ ciclo do PMAQ-AB (Quadro 1). 
A Tabela 1 mostra que na Região Norte, a maioria dos cirurgiões dentistas $(74,3 \%)$ atuava na ESB por um período de até 03 anos de serviço. Nos estados de Roraima $(65,0 \%)$ e Tocantins $(37,4 \%)$, a maioria dos CD atuava nas ESB por até 01 ano, enquanto nos demais Estados da Região, o predomínio é de CD com atuação na ESB entre 01 a 03 anos. Em contrapartida, ao se analisar o quantitativo de CD com atuação na ESB por mais de 10 anos, apenas $35 \mathrm{CD}(2,94 \%)$ se encontrava nessa condição, com destaque para os estados do Amapá e Roraima, os quais não possuíam nenhum $\mathrm{CD}$ atuando por mais de 10 anos na ESB.

Quanto ao vínculo empregatício, $573(48,1 \%)$ dos CD da Região Norte se encontravam no serviço de forma temporária (administração pública ou prestação de serviço), em que o estado do Amapá se destacou pela presença de 83,1\% de seus CD nesse vínculo, porém esse estado foi o que apresentou menor percentual $(3,4 \%)$ de servidores públicos estatutários, quando comparado aos demais estados da região. O estado do Tocantins, quando analisado isoladamente, foi o que mais apresentou CD $(5,1 \%)$ com vínculo de Contrato CLT (Tabela 1).

Ao se analisar a forma de ingresso do CD na ESB, observou-se que na Região Norte, que predominou o concurso público $(42,4 \%)$, sendo essa a forma de ingresso da maioria dos CD nos Estados de Rondônia $(85,9 \%)$, Acre $(65,0 \%)$ e Tocantins $(51,4 \%)$. Constatou-se que nos Estados do
Amapá, Amazonas e Pará, esse ingresso para atuação nas ESB ocorreu de forma majoritária por indicação (Tabela 1).

No que se refere a formação acadêmica, constatou-se que a maioria dos CD atuantes na ESB da Região Norte não possuem qualquer formação em nível lato sensu ou stricto sensu. Dos que possuíam, $12,0 \%$ apresentaram formação em nível de especialização/ residência em saúde coletiva/saúde pública e $10,1 \%$ saúde da família. Observou-se que o estado do Pará foi o que apresentou o menor percentual de CD com formação em Saúde Pública ou Saúde da Família quando comparado com os demais estados da Região Norte (Tabela $1)$.

Na Tabela 2 verifica-se que nos 07 estados da Região Norte o CD participa de ações de Educação Permanente em Saúde (EPS), com destaque para os Estados do Amazonas (79,8\%) e Tocantins (74,7\%).

Dentre as ações, observou-se que os seminários, mostras, oficinas e grupos de discussão foram os mais frequentes em todos os estados da Região. Contudo, os cursos de educação a distância e o telessaúde ainda aparecem de forma tímida entre as ações de EPS (Gráfico 1).

Observou-se que os CD, da maioria dos Estados da Região, consideram que as ações de EPS contemplam as necessidades da ESB. Contudo, no estado do Amapá constatou-se que a carência de ações de EPS ofertadas à ESB faz com que os CD considerem que as ações não atendem às necessidades da equipe (Tabela 2). 
Tabela 1: Perfil do cirurgião dentista que atua nas Equipes de Saúde Bucal no Pará. PMAQ-AB, 2014.

\begin{tabular}{|c|c|c|c|c|c|c|c|c|c|c|c|c|c|c|c|c|}
\hline & \multicolumn{2}{|c|}{$\mathbf{A C}$} & \multicolumn{2}{|c|}{$\mathbf{A P}$} & \multicolumn{2}{|c|}{$\mathbf{A M}$} & \multicolumn{2}{|c|}{ PA } & \multicolumn{2}{|c|}{ RO } & \multicolumn{2}{|c|}{$\mathbf{R R}$} & \multicolumn{2}{|c|}{ TO } & \multicolumn{2}{|c|}{ TOTAL } \\
\hline & $\mathbf{n}$ & $\%$ & $\mathbf{n}$ & $\%$ & $\mathbf{n}$ & $\%$ & $\mathbf{n}$ & $\%$ & $\mathbf{n}$ & $\%$ & $\mathbf{n}$ & $\%$ & $\mathbf{n}$ & $\%$ & $\mathbf{n}$ & $\%$ \\
\hline \multicolumn{17}{|l|}{ Tempo de atuação na ESB } \\
\hline Menos de 1 ano & 10 & 16,7 & 15 & 25,4 & 89 & 33,3 & 135 & 34,5 & 24 & 17,8 & 13 & 65,0 & 96 & 37,4 & 382 & 32,1 \\
\hline De 1 a 3 anos & 31 & 51,7 & 23 & 39,0 & 130 & 48,7 & 158 & 40,4 & 61 & 45,2 & 7 & 35,0 & 92 & 35,8 & 502 & 42,2 \\
\hline De 4 a 6 anos & 7 & 11,7 & 17 & 28,8 & 32 & 12,0 & 59 & 15,1 & 31 & 23,0 & 0 & 0 & 27 & 10,5 & 173 & 14,5 \\
\hline De 7 a 9 anos & 8 & 13,3 & 4 & 6,8 & 13 & 4,9 & 29 & 7,4 & 12 & 8,9 & 0 & 0 & 31 & 12,1 & 97 & 8,1 \\
\hline Mais de 10 anos & 4 & 6,7 & 0 & 0 & 3 & 1,1 & 10 & 2,6 & 7 & 5,2 & 0 & 0 & 11 & 4,3 & 35 & 2,94 \\
\hline \multicolumn{17}{|l|}{ Tipo de vínculo } \\
\hline Servidor público estatutário & 25 & 41,7 & 2 & 3,4 & 88 & 33,0 & 120 & 30,7 & 111 & 82,2 & 8 & 40,0 & 127 & 49,4 & 481 & 40,4 \\
\hline Cargo comissionado & 0 & 0 & 2 & 3,4 & 4 & 1,5 & 1 & 0,3 & 5 & 3,7 & 2 & 10,0 & 4 & 1,6 & 18 & 1,5 \\
\hline Temporário - administração pública & 8 & 13,3 & 32 & 54,3 & 88 & 33,0 & 71 & 18,2 & 5 & 3,7 & 5 & 25,0 & 53 & 20,6 & 262 & 22,0 \\
\hline Temporário - prestação de serviço & 11 & 18,3 & 17 & 28,8 & 65 & 24,3 & 167 & 42,7 & 1 & 0,7 & 0 & 0 & 50 & 19,5 & 311 & 26,1 \\
\hline Empregado público CLT & 13 & 21,7 & 0 & 0 & 2 & 0,8 & 2 & 0,5 & 8 & 5,9 & 0 & 0 & 4 & 1,6 & 29 & 2,43 \\
\hline Contrato CLT & 1 & 1,7 & 1 & 1,7 & 11 & 4,1 & 15 & 3,8 & 5 & 3,7 & 1 & 5,0 & 13 & 5,1 & 47 & 3,95 \\
\hline Outros & 0 & 0 & 4 & 6,8 & 5 & 1,9 & 13 & 3,3 & 0 & 0 & 1 & 5,0 & 6 & 2,3 & 29 & 2,43 \\
\hline Não sabe/ não respondeu & 2 & 3,3 & 1 & 1,7 & 4 & 1,5 & 2 & 0,5 & 0 & 0 & 3 & 15,0 & 0 & 0 & 12 & 1,0 \\
\hline \multicolumn{17}{|l|}{ Tipo de ingresso no serviço } \\
\hline Concurso público & 39 & 65,0 & 1 & 1,7 & 86 & 32,2 & 123 & 31,5 & 116 & 85,9 & 8 & 40,0 & 132 & 51,4 & 505 & 42,4 \\
\hline Seleção pública & 5 & 8,3 & 15 & 25,4 & 58 & 21,7 & 41 & 10,5 & 14 & 10,4 & 2 & 10,0 & 9 & 3,5 & 144 & 12,1 \\
\hline Indicação & 8 & 13,3 & 33 & 55,9 & 95 & 35,6 & 152 & 38,9 & 4 & 3,0 & 8 & 40,0 & 65 & 25,3 & 365 & 30,6 \\
\hline Outra forma & 8 & 13,3 & 10 & 16,9 & 28 & 10,5 & 75 & 19,1 & 1 & 0,7 & 2 & 10,0 & 51 & 19,9 & 175 & 14,7 \\
\hline \multicolumn{17}{|l|}{ Formação acadêmica } \\
\hline \multicolumn{17}{|l|}{ Especialização/ Residência } \\
\hline Saúde da Família & 9 & 15,0 & 9 & 15,3 & 24 & 8,9 & 23 & 5,9 & 17 & 12,6 & 6 & 30,0 & 33 & 12,8 & 121 & 10,1 \\
\hline Saúde Coletiva/ Saúde Pública & 5 & 8,4 & 7 & 11,9 & 38 & 14,2 & 26 & 6,6 & 25 & 18,5 & 3 & 15,0 & 39 & 15,2 & 143 & 12,0 \\
\hline Outra & 8 & 13,3 & 4 & 6,7 & 31 & 11,8 & 29 & 7,4 & 14 & 10,4 & 4 & 20,0 & 29 & 11,3 & 119 & 10,0 \\
\hline Não possui & 38 & 63,3 & 39 & 66,1 & 174 & 65,1 & 313 & 80,1 & 79 & 58,5 & 7 & 35,0 & 156 & 60,7 & 806 & 67,7 \\
\hline \multicolumn{17}{|l|}{ Mestrado } \\
\hline Saúde da Família & 3 & 5,0 & 0 & 0 & 2 & 0,8 & 1 & 0,3 & 0 & 0 & 1 & 5 & 0 & 0 & 7 & 0,5 \\
\hline Saúde Coletiva/ Saúde Pública & 2 & 3,3 & 0 & 0 & 2 & 0,8 & 3 & 0,8 & 3 & 2,2 & 1 & 5 & 2 & 0,8 & 13 & 1,09 \\
\hline
\end{tabular}

71 


\section{Tabela 1. (cont.)}

\begin{tabular}{|c|c|c|c|c|c|c|c|c|c|c|c|c|c|c|c|c|}
\hline Outra & 2 & 3,3 & 10 & 17 & 10 & 3,7 & 15 & 3,8 & 12 & 8,9 & 2 & 10 & 12 & 4,7 & 63 & 5,29 \\
\hline Não possui & 53 & 88,4 & 49 & 83 & 253 & 94,7 & 372 & 95,1 & 120 & 88,9 & 16 & 80 & 243 & 94,5 & 1106 & 93,0 \\
\hline \multicolumn{17}{|l|}{ itorado } \\
\hline Saúde da Família & 1 & 1,7 & 0 & 0 & 1 & 0,4 & 2 & 0,5 & 1 & 0,7 & 1 & 5 & 0 & 0 & 6 & 0,5 \\
\hline Saúde Coletiva/ Saúde Pública & 1 & 1,7 & 0 & 0 & 0 & 0 & 1 & 0,3 & 0 & 0 & 1 & 5 & 0 & 0 & 3 & 0,2 \\
\hline Outra & 3 & 5,0 & 2 & 3,4 & 3 & 1,1 & 2 & 0,5 & 2 & 1,5 & 0 & 0 & 2 & 0,8 & 14 & 1,17 \\
\hline Não possui & 55 & 91,6 & 57 & 96,6 & 263 & 98,5 & 386 & 98,7 & 132 & 97,8 & 18 & 90 & 255 & 99,2 & 1166 & 98 \\
\hline l & 60 & 100 & 59 & 100 & 267 & 100 & 391 & 100 & 135 & 100 & 20 & 100 & 257 & 100 & 1189 & 100 \\
\hline
\end{tabular}

Fonte: PMAQ-AB, 2014.

Tabela 2: Ações de Educação Permanente em que o cirurgião dentista se insere na Estratégia Saúde da Família. PMAQ-AB, 2014.

\begin{tabular}{|c|c|c|c|c|c|c|c|c|c|c|c|c|c|c|c|c|}
\hline & \multicolumn{2}{|c|}{ AC } & \multicolumn{2}{|c|}{$\mathbf{A P}$} & \multicolumn{2}{|c|}{$\mathbf{A M}$} & \multicolumn{2}{|c|}{ PA } & \multicolumn{2}{|c|}{ RO } & \multicolumn{2}{|c|}{$\mathbf{R R}$} & \multicolumn{2}{|c|}{ TO } & \multicolumn{2}{|c|}{ TOTAL } \\
\hline & $\mathbf{n}$ & $\%$ & $\mathbf{n}$ & $\%$ & $\mathbf{n}$ & $\%$ & $\mathbf{n}$ & $\%$ & $\mathbf{n}$ & $\%$ & $\mathbf{n}$ & $\%$ & $\mathbf{n}$ & $\%$ & $\mathbf{n}$ & $\%$ \\
\hline \multicolumn{17}{|c|}{ Participa de ações de educação permanente } \\
\hline Sim & 33 & 55,0 & 40 & 67,8 & 213 & 79,8 & 243 & 62,1 & 94 & 69,6 & 13 & 65,0 & 192 & 74,7 & 828 & 69,6 \\
\hline Não & 27 & 45,0 & 19 & 32,2 & 54 & 20,2 & 148 & 37,9 & 41 & 30,4 & 7 & 35,0 & 65 & 25,3 & 361 & 30,3 \\
\hline \multicolumn{17}{|c|}{ As ações atendem as necessidades da ESB? } \\
\hline Sim & 16 & 26,7 & 17 & 28,8 & 125 & 46,8 & 155 & 39,6 & 51 & 37,8 & 10 & 50,0 & 149 & 58,0 & 523 & 43,9 \\
\hline Não & 17 & 28,3 & 23 & 39,0 & 88 & 33,0 & 88 & 22,5 & 43 & 31,9 & 3 & 15,0 & 43 & 16,7 & 305 & 25,6 \\
\hline Não sabe/não respondeu & 27 & 45,0 & 19 & 32,2 & 54 & 20,2 & 148 & 37,8 & 41 & 30,4 & 7 & 35,0 & 65 & 25,3 & 361 & 30,3 \\
\hline Total & 60 & 100 & 59 & 100 & 267 & 100 & 391 & 100 & 135 & 100 & 20 & 100 & 257 & 100 & 1189 & 100 \\
\hline
\end{tabular}

Fonte: PMAQ-AB, 2014. 


\section{Discussão}

O maior campo de atuação do cirurgião dentista no serviço público foi fortemente influenciado pela inserção do CD nas Equipes de Saúde Bucal das Estratégias Saúde da Família. Para isso, se faz necessária, a adequação da formação desses profissionais saindo de um campo individual, tecnicista e de práticas de consultório, para a atuação em equipes multiprofissionais, integrados com a comunidade assistida, conduzindo ações de promoção de saúde e produzindo conhecimento a partir do seu próprio posto de trabalho e necessidades da comunidade.

Nesse enfoque, é importante conhecer o perfil dos profissionais da ESB, o qual está vinculado a características pessoais, aos seus processos de formação e qualificação, e de suas experiências, vivências e práticas. Esse perfil pode ser aperfeiçoado com a implementação de estratégias de EPS, entre outras ações que valorizam o profissional ${ }^{15}$.

Por meio do Instrumento de Avaliação Externa para as Equipes de Saúde Bucal, que compõe a terceira fase do PMAQ-AB, os profissionais da ESB foram entrevistados, com o objetivo de se obter informações a respeito do processo de trabalho da equipe e a organização do serviço e do cuidado com os pacientes ${ }^{13}$.

Algumas políticas do MS, como o Programa de Valorização dos Profissionais da Atenção Básica (PROVAB), que possuía a intenção de atrair profissionais para laborar na Atenção Básica, incentivou a presença de trabalhadores recém-formados e com pouca experiência ${ }^{16}$. Contudo, estudos mostram que o PROVAB foi criticado por mobilizar profissionais para atuarem na Atenção Básica de municípios com déficit de profissionais e com grande dificuldade de fixação ${ }^{17}$. Nesta pesquisa, de acordo com a Tabela 1, observou-se que, a maioria dos CD da Região Norte atuava na ESB de 01 a 03 anos (42,2\%), o que pode ter sido favorecido pela oferta de vagas à incorporação de cirurgiões dentistas pelo PROVAB em 2013.

Uma pesquisa realizada pelo $\mathrm{MS}^{18}$ demonstrou que os vínculos trabalhistas deficitários estabelecidos com os profissionais das equipes de saúde contribuem para alta rotatividade e insatisfação profissional. Além disso, destacou que o pequeno tempo de permanência dos profissionais nas equipes pode se constituir em fator limitante para o trabalho, pois dificulta a qualificação dos profissionais e o desempenho das ações, visto que perdem a oportunidade de aderir e incorporar novos valores e exercitar novas práticas de saúde. Nesse contexto, ressaltase também a dificuldade de se estabelecer vínculos com a comunidade assistida.

Quanto ao tipo de vínculo do CD na ESB, o MS recomenda que a contratação dos profissionais para a ESF, além da opção de seleção interna (na qual se utiliza indivíduos que já trabalham na prefeitura), também necessita de profissionais de saúde que não pertencem aos quadros municipais, por meio de contratação pelo regime da Consolidação das Leis do Trabalho (CLT), pois por meio dessa via ocorrerá expansão e reposição do quadro de pessoal da administração pública nos três níveis de governo, observados os limites de despesas estabelecidos pela Lei de Responsabilidade Fiscal ${ }^{19}$. Porém, ao serem analisados os resultados do presente estudo (Tabela 1), pôde-se observar que de 1.189 CD entrevistados, apenas $47(3,95 \%)$ são vinculados pela modalidade 'contrato $C L T$ ', inferindo-se que, de alguma forma, as demais modalidades de vínculo profissional podem ter onerado os limites de despesas pré-estabelecidos, principalmente nos estados do Acre, Amapá e Roraima, que apresentaram menos CD com esse tipo de vínculo.

Durante estudo realizado pelo Núcleo de Pesquisa em Saúde Coletiva (NESCON/ UFMG) juntamente com a Coordenação Geral da Política de Recursos Humanos do MS, verificou-se que na região Sudeste os CD foram contratados por 
vínculos temporários ou prestação de serviços em $56,4 \%$ dos casos $^{20}$. A realidade encontrada nos resultados analisados do Norte do país mostrou que as ESB no estado do Pará foram as que mais apresentaram o tipo de vínculo 'temporário-prestação de serviço' (Tabela 1). Isso demonstra que os municípios além de não resguardarem os direitos trabalhistas dos profissionais, fazem com que estes fiquem à mercê das mudanças políticas ${ }^{21}$.

Palú $^{22}$ relatou que os CD contratados temporariamente acabam se submetendo às mudanças políticas, buscando outros vínculos empregatícios ou fontes de renda, deixando de se dedicar à mudança do modelo de atenção à saúde para a conquista de melhores resultados no serviço e nos indicadores de saúde da população. Estudo realizado com $\mathrm{CD}$ da ESF no estado do Ceará demonstrou que os padrões salariais bastante defasados e a falta de vínculo empregatício incomodavam os profissionais a ponto de intervir em suas práticas $^{23}$.

A maioria dos contratos nas Equipes de Saúde da Família no estado do Pará eram temporários, e a maior forma de seleção dos trabalhadores foi por meio de indicação. Isso gera implicação no trabalho dessas equipes, pois contratos que podem ser encerrados a qualquer momento não oferecem estabilidade, fortalecendo a rotatividade nas equipes, bem como fazendo com que seus integrantes sejam substituídos constantemente, comprometendo o processo de vínculo com a comunidade.

A forma de ingresso na ESF é importante, pois é um dos elementos que caracteriza o conhecimento e o envolvimento do profissional com essa nova proposta de atuação em saúde. Os CD que são inseridos sem o devido preparo para esse novo modelo de atenção à saúde, acabam iniciando o trabalho de forma inadequada, podendo comprometer os resultados positivos junto à estratégia ${ }^{22}$. A seleção de profissionais para atuar na Estratégia Saúde da Família deve estar regida pela legislação vigente, e baseada nas orientações do $\mathrm{MS}^{24}$.

Koster e Machado ${ }^{25}$ mostraram que o MS apresentou em suas políticas de Gestão do Trabalho no SUS o posicionamento de que a única maneira legal de não fortalecer a precarização do trabalho no SUS é o ingresso por meio de concurso público e de processo seletivo público. Analisando os resultados obtidos na região Norte do país, foi possível perceber que o concurso público foi a principal forma de ingresso do CD $(42,4 \%)$ no serviço, apesar dessa forma não ser a predominante nos estados do Amapá, Amazonas e Pará (Tabela 1).

Em contrapartida, as indicações são métodos rotineiros de contrato em alguns municípios para o trabalho na $\mathrm{ESF}^{26}$. Isso pode contribuir para a inserção de profissionais sem experiência de atuação na Atenção Básica e na proposta de cuidado da ESF, bem como incorre que a constante troca de gestão durante os mandatos influencia para a insegurança dos contratos de trabalho e o comprometimento na longitudinalidade do cuidado necessário à ESF. Os resultados desta pesquisa mostraram que $30,6 \%$ dos $\mathrm{CD}$ da região ingressaram na ESB por indicação, sendo 55,9\% no Estado do Amapá, 35,6\% no Amazonas e 38,9\% no Pará, estados onde essa é a principal forma de ingresso (Tabela $1)$.

No que se refere a formação do $\mathrm{CD}$, os resultados mostraram que a maioria não possuía nem estava cursando nenhum curso de pós-graduação em nível lato sensu ou stricto sensu (Tabela 1). Costa et al. ${ }^{27}$ comentaram sobre a necessidade da formação dos profissionais de saúde com níveis mais elevados de educação e qualificação, pois assim, podem estar mais capacitados para atuação em saúde coletiva e comunitária.

Nesse contexto, ressalta-se que muitos profissionais da pesquisa atuavam no interior do estado, estando em posição de desvantagem em relação aos profissionais que trabalhavam na capital ou próxima a 
ela, visto a maior dificuldade de acesso aos cursos de atualização e capacitação profissional, principalmente devido à extensão territorial e à extensa bacia hidrográfica de alguns estados da Região Norte. Martins ${ }^{28}$ evidenciou em seu estudo que $C D$ que trabalhavam no interior do Estado do Amazonas mencionaram dificuldades de realizar cursos de pósgraduação, devido gastos com deslocamentos e resistência da gestão local. Sobre o assunto, Cutolo ${ }^{29}$ destaca a importância da formação e sensibilização dos gestores, visto que, não adiantaria investimento em recursos humanos, se os administradores, ordenadores e políticos não tiverem compromisso com os pilares do SUS.

Nesse enfoque, o aprendizado por meio de recursos tecnológicos, para atualização de profissionais, deve ser valorizado como uma estratégia, sobretudo aos profissionais que residem e trabalham mais distantes dos centros urbanos. Contudo, observou-se que $90 \%$ dos CD da região não participavam de cursos de educação à distância (Gráfico 1). Essa modalidade de instrução permite maior flexibilidade e abre uma janela de acesso ao conhecimento e à informação, possuindo forma oportuna, personalizada e dinâmica, em comparação com as atividades de ensino presencial ${ }^{15}$. No entanto, as dificuldades de acesso à internet em municípios mais distantes, e a pouca disseminação das Tecnologias de Informação e Comunicação (TIC) virtuais nas áreas mais longínquas das capitais da região, podem ter influenciado o resultado encontrado neste estudo. Dessa forma, é necessário fortalecer os modelos educativos à distância, priorizando a problematização e incorporando-os ao desenvolvimento de projetos de EPS ${ }^{15}$.

Os resultados obtidos neste estudo mostraram que a maioria dos CD que atuam nos estados da região Norte do Brasil, participavam de ações de EPS nas ESB que pertenciam, e na maioria dos estados da região, estas atendiam as necessidades da ESB (Tabela 2). Reconhecer a importância da EPS é competência dos municípios e das unidades de saúde, devendo fortalecer ações que possam conciliar necessidades e possibilidades singulares da demanda. É importante encontrar o equilíbrio entre as ofertas de EPS pré-formatadas, com o momento e contexto das equipes, para que possam ser aproveitadas com efetividade, e de modo produtivo e contínuo pelos profissionais $^{30}$.

Dentre as estratégias de EPS mais utilizadas pelos CD da Região Norte estão os seminários, mostras, oficinas e grupos de discussão, seguidos pela troca de experiências. O Telessaúde e as atividades promovidas pelo Sistema Universidade Aberta do SUS (UNASUS) ainda apareceram de forma tímida nos estados da Região (Gráfico 1). Isso identifica que ainda persiste nos serviços de saúde, estratégias de educação conservadoras, com metodologias de transmissão tradicionais ${ }^{31}$. Fato semelhante também foi registrado em estudo com trabalhadores no estado de Goiás, demonstrando dificuldades de compreensão da EPS por parte dos atores envolvidos, enfatizando a necessidade de novas ações de EPS para promover a reflexão e ampliação do trabalho em saúde, bem como subsidiar o planejamento estratégico, a construção de planos e o apoio às instâncias responsáveis pelo fomento e gerenciamento da EPS nos municípios, para fortalecimento dos serviços de saúde ${ }^{32}$.

Ceccim e Feuerwerker ${ }^{33}$ afirmaram que a formação para a área da saúde deveria ter como foco a transformação das práticas profissionais e da própria organização do trabalho. A ótica da formação, principalmente da EPS, é que essa se fortaleça, despertando a sua capacidade de acolher e cuidar da saúde das populações do seu território. Contudo, o processo de trabalho fragmentado, em que cada profissional atua na sua área, com conhecimentos isolados uns dos outros, promove dificuldades de interação entre membros das equipes de saúde ${ }^{31}$.

Ferla et al. ${ }^{34}$ relataram a gestão da aprendizagem no cotidiano do trabalho, 
como sendo aquela que gera o desenvolvimento do trabalho e não apenas a qualificação do trabalhador. Nesse enfoque, o incentivo e apoio dos gestores são de grande importância para o desenvolvimento das propostas de EPS, visto que é um instrumento que requer reflexão crítica, pois se não há um gestor sensibilizado com a proposta, podem ocorrer conflitos que atrapalham uma ação sólida com resultados satisfatórios $^{35}$. Neste sentido, o PMAQ-AB, enquanto programa indutor de mudanças, por meio de suas avaliações cíclicas, permite reflexões na equipe de saúde para a importância da EPS para o trabalho em saúde ${ }^{36}$.

\section{Conclusão}

A maioria dos CD inserido nas ESB da Região Norte do Brasil era servidor público temporário e que participava das ações de educação permanente na ESF que integrava.

Os estados do Amapá, Amazonas e Pará mostraram maior percentual de CD com vínculo temporário, tendo como consequência falta de estabilidade no serviço, maior rotatividade nas equipes e fragilidade nos vínculos do sistema de saúde.

A participação em ações de EPS foi detectada em todos os estados da região norte do país. As ações que se destacaram foram as estratégias tradicionais $\mathrm{e}$ presenciais, com destaque para os seminários, mostras, oficinas e grupos de discussão.

Dessa forma, é importante que os atores envolvidos no sistema de saúde entendam que a EPS não visa somente a qualificação profissional, mas sim proporciona a transformação das práticas no cotidiano dos serviços de saúde. Além disso, aspectos como a forma de ingresso e o tipo de vínculo do $\mathrm{CD}$ geram fragilidades em sua atuação e comprometimento dos vínculos tanto com a equipe multidisciplinar, quanto com a comunidade em que os profissionais atuam.

\section{Referências}

1. Bomfim ES, Oliveira BG, Rosa RS, Almeida MVG, Silva SS, Araújo IB. Educação permanente no cotidiano das equipes de saúde da família: utopia, intenção ou realidade? Rev Fund Care Online. 2017; 9(2):526-35.

2. Brasil. Ministério da Saúde. Secretaria de Atenção à Saúde. Departamento de Atenção Básica. Autoavaliação para Melhoria do Acesso e da Qualidade da Atenção Básica (AMAQ): $2^{\mathrm{a}}$ ed. Brasília: Ministério da Saúde, 2016. 180p.

3. Brasil. Ministério da Saúde. Secretaria de Atenção à Saúde. Departamento de Atenção Básica. Diretrizes da Política Nacional de Saúde Bucal. Brasília: Ministério da Saúde, 2004. $16 \mathrm{p}$.

4. Brasil. Ministério da Saúde. Portaria GM/MS no 198, de 13 de fevereiro de 2004. Institui a Política Nacional de Educação Permanente em Saúde como estratégia do Sistema Único de Saúde para a formação e o desenvolvimento de trabalhadores para o setor e dá outras providências. Brasília: Ministério da Saúde, 2004.

5. Brasil. Ministério da Saúde. Portaria GM/MS n ${ }^{\circ} 1.996$, de 20 de agosto de 2007. Dispõe sobre as diretrizes para a implementação da Política Nacional de Educação Permanente em Saúde e dá outras providências. Brasília: Ministério da Saúde, 2007.

6. Ceccim RB, Ferla AA. Educação e saúde: ensino e cidadania como travessia de fronteiras. Trab Educ Saúde 2009; 6(3):443-56. 
7. Ferreira L, Barbosa JSA, Esposti CDD, Cruz MM. Educação Permanente em Saúde na atenção primária: uma revisão integrativa da literatura. Saúde Debate 2019; 43(120): 223 239.

8. Maciel JAC, Vasconcelos MIO, Castro-Silva II, Eloia SMC, Farias MR. Educação permanente em saúde para o cirurgião-dentista da Estratégia Saúde da Família: Uma revisão integrativa. Rev APS 2017; 20(3): 414-22.

9. Brasil. Ministério da Saúde. Portaria n ${ }^{\circ}$ 1654, de 19 de julho de 2011. Institui, no âmbito do Sistema Único de Saúde, o Programa Nacional de Melhoria do Acesso e da Qualidade da Atenção Básica (PMAQ-AB) e o Incentivo Financeiro do PMAQ-AB, denominado Componente de Qualidade do Piso da Atenção Básica Variável-PAB variável. Brasília: Ministério da Saúde, 2011.

10. Brasil. Ministério da Saúde. Saúde Mais Perto de Você - Acesso e Qualidade - Programa de Melhoria do Acesso e da Qualidade: Documento Síntese para Avaliação Externa. Brasília: Ministério da Saúde, 2012. 55p.

11. Ribeiro DT, Nascimento DT, Cunha FM, Ozorio JC, Ferreira AV, Santos TC, et al. O PMAQ-AB como umas das estratégias de estímulo à prática da Educação Permanente em Saúde. In: Gomes LB, Barbosa MG, Ferla AA (Org.). A educação permanente em saúde e as redes colaborativas: conexões para a produção de saberes e práticas, 2016. p. 129-41.

12. Morita MC, Haddad AE, Araújo ME. Perfil atual e tendências do cirurgião-dentista brasileiro. Maringá: Dental Press, 2010. 96p.

13. Brasil. Ministério da Saúde. Secretaria de Atenção à Saúde. Departamento de Atenção Básica. Manual instrutivo para as equipes de atenção básica (Saúde da Família, Saúde Bucal e Equipes Parametrizadas) E NASF. Brasilia, 2013. 38p.

14. Brasil. Ministério da Saúde. Secretaria de Atenção à Saúde. Departamento de Atenção Básica. Instrumento de avaliação externa de saúde mais perto de você - acesso e qualidade. Brasilia-DF, 2013. 50p.

15. Brasil. Ministério da Saúde. Secretaria de Atenção à Saúde. Departamento de Gestão da Educação em Saúde. Política Nacional de Educação Permanente em Saúde. Brasília: Ministério da Saúde, 2009. 64p.

16. Fontenelle LF. Mudanças recentes na Política Nacional de Atenção Básica: uma análise crítica. Rev Bras Med Fam Comunidade 2012; 7(22):5-9.

17. Brasil. Ministério da Saúde. Gabinete do Ministro. Portaria Interministerial no 2.087 , de 01 de setembro de 2011. Institui o Programa de Valorização do Profissional da Atenção Básica. Diário Oficial da República Federativa do Brasil. Brasília: Ministério da Saúde, 2011.

18. Brasil. Ministério da Saúde. Secretaria de Atenção à Saúde. Departamento de Atenção Básica. Avaliação normativa do Programa Saúde da Família no Brasil: monitoramento da implantação e funcionamento das equipes de saúde da família: 2001-2002. Brasília: Ministério da Saúde, 2004. 140p.

19. Brasil. Ministério da Saúde. Guia prático do Programa Saúde da Família. Brasília: Ministério da Saúde; 2001. 67p.

20. Girardi SN, Carvalho CL, Girardi JB, Araújo JF. Configurações do mercado e trabalho dos assalariados em saúde no Brasil. In: Observatório de Recursos Humanos em Saúde no Brasil: estudos e análises. Brasília: Ministério da Saúde, 2004. p.121-37.

21. Lourenço EC, Silva ACB, Meneghin MC, Pereira AC. A inserção de equipes de saúde bucal no Programa Saúde da Família no Estado de Minas Gerais. Ciênc Saúde Colet 2009; 14(Supl. 1):1367-77.

22. Palú APN. A inserção da saúde bucal no PSF, perspectivas e desafios: a visão de odontólogos do Paraná. [Dissertação] Mestrado em Saúde Coletiva. Universidade Estadual de Londrina-PR. 2004. 103p.

23. Facó EF, Viana LMO, Bastos VA, Nuto SAS. O Cirurgião-Dentista e o programa saúde da família na microrregião II, Ceará, Brasil. Rev Bras Promoç Saúde 2005; 18(2):70-7. 
24. Mafra LAS, Vilela EM. Estratégia Saúde da Família: Contratação Temporária e Precarização nas Relações de Trabalho. Cad Estudos Interdiscipl 2015; (Gestão Pública e Sociedade): $38-52$.

25. Koster I, Machado MHA. Gestão do trabalho e o contexto da flexibilização no Sistema Único de Saúde. Divulg Saúde Debate 2012: 33-44.

26. Alvarenga EC, Oliveira PTR, Pinheiro HHC, Carneiro VCCB. Condições de trabalho de equipes de saúde da família do Pará. Rev Nufen: Phenom Interd 2018; 10(1):58-72.

27. Costa RM, Medeiros-Junior A, Costa ICC, Pinheiro IVA. O trabalho em equipe desenvolvido pelo cirurgião-dentista na Estratégia Saúde da Família: expectativas, desafios e precariedades. Rev Bras Med Fam Comunidade 2012; 7(24):147-63.

28. Martins FM. Educação Permanente em Saúde no interior do Estado do Amazonas: Estudo de Caso na Região de Saúde Rio Madeira/AM. [Dissertação] Universidade Federal do Amazonas. Amazonas. 2015.

29. Cutolo LRA. O SUS e a Formação de Recursos Humanos. ACM: Arq Catarin Med 2003; 32(2):49-59.

30. Brasil. Ministério da Saúde. Secretaria de Atenção à Saúde. Departamento de Atenção Básica. Política Nacional de Atenção Básica. Brasília: Ministério da Saúde, 2012. 110p.

31. Franco TB. Produção do cuidado e produção pedagógica: integração de cenários do sistema de saúde no Brasil. Interface (Botucatu) 2007; 11(23):427-38.

32. Barcellos RMS, Melo LM, carneiro LA, Souza AC, Lima DM, Rassi LT. Educação permanente em saúde: práticas desenvolvidas nos municípios do estado de Goiás. Trab Educ Saúde 2020; 18(2): e0026092.

33. Ceccim RB, Feuerwerker LCM. O quadrilátero da formação para a área da saúde: ensino, gestão, atenção e controle social. Physis 2004; 14(1):41-65.

34. Ferla AA, Rocha CMF, Dias MTG, Santos LM. Redes vivas de Educação e Saúde e a Integração Universidade e Sistema Local de Saúde: saberes locais e múltiplas saúdes como capacidade profissional e como atributo das Redes de Atenção. In: Ferla AA, Rocha CMF, Dias MTG, Santos LM (Org.). Redes Vivas de Educação e Saúde: Relatos e vivências da universidade e sistemas de saúde. Porto Alegre: Rede UNIDA, 2015. p. 9-21.

35. Amorim ACM. Educação Permanente na Estratégia de Saúde da Família: oportunidades de aprendizagem e inovação da prática profissional. [Dissertação] Mestrado Profissional. Universidade Federal de São Paulo. Programa de Pós-Graduação Ensino em Ciências da Saúde. São Paulo. 2013.

36. Almeida TMC, Santos RMM, Sampaio DMN, Vilela ABA. Planejamento e desenvolvimento de ações de Educação Permanente em Saúde na perspectiva do PMAQAB. Saúde Debate 2019; 43(Spec 1): 77-85.

\section{Como citar este artigo:}

Emmi DT, Lima ZS, Miranda MSL. Perfil do cirurgião dentista das equipes de saúde bucal e inserção em ações de educação permanente na Região Norte do Brasil. Rev. Aten. Saúde. 2021; 19(67): 66-78. 